TITLE:

\title{
Urban-Area Extraction From Polarimetric SAR Images Using Polarization Orientation Angle
}

$\operatorname{AUTHOR}(S):$

Kajimoto, M.; Susaki, J.

\section{CITATION:}

Kajimoto, M. ... [et al]. Urban-Area Extraction From Polarimetric SAR Images Using

Polarization Orientation Angle. IEEE Geoscience and Remote Sensing Letters 2013, 10(2): 337-341

ISSUE DATE:

2013-03

URL:

http://hdl.handle.net/2433/193301

\section{RIGHT:}

(c) 2013 IEEE. Personal use of this material is permitted. Permission from IEEE must be obtained for all other uses, in any current or future media, including reprinting/republishing this material for advertising or promotional purposes, creating new collective works, for resale or redistribution to servers or lists, or reuse of any copyrighted component of this work in other works.; この論文は出版社版でありません。引用の際には出版社版をご確認ご利用ください。; This is not the published version. Please cite only the published version. 


\title{
Urban Area Extraction from Polarimetric SAR Images Using Polarization Orientation Angle
}

\author{
Muneyoshi Kajimoto and Junichi Susaki, Member, IEEE
}

\begin{abstract}
In this paper, an algorithm is proposed that robustly extracts urban areas from polarimetric synthetic aperture radar (SAR) images. Polarization orientation angle (POA), volume scattering power $(\mathrm{Pv})$ derived by four-component decomposition, and total power (TP) are utilized in the proposed algorithm. The dependence of the four decomposition components on POA can be lessened by rotating the elements of the coherency matrix by the POA. However, a level of POA dependence remains even after the correction. The proposed algorithm utilizes POA-corrected components, but pixels are grouped into several categories according to POA. First, urban and farmland training data are selected for each category in a study area. Then, urban and mountain areas are separated from farmland, bare ground, and sea by utilizing the Pv-TP scattergram. Finally, a measure of the POA randomness between neighboring pixels is used to discriminate between urban areas with nearly homogeneous POA and mountain areas with randomly distributed POAs. When performing classification on more than one study area, thresholds manually selected for one of the study areas are used to automatically estimate thresholds for the other areas. An accuracy assessment demonstrates that POA-based categorization and utilization of POA randomness contribute to improving classification accuracy.
\end{abstract}

Index Terms-Four-component decomposition, polarimetric synthetic aperture radar, polarization orientation angle, urban area extraction.

\section{INTRODUCTION}

$\mathrm{L}$ AND cover classification is a highly anticipated $\longrightarrow$ application of satellite remote sensing. Land cover classification using synthetic aperture radar (SAR) has proved difficult, however, because the scattering of microwaves emitted from SAR has a greater dependence on target structure than on land cover type. Even in urban areas, a large change in scattering intensity is found, dependent on whether the normal to a building's wall is parallel to the sensor's ground range direction. When this normal is parallel to the range direction, the scattering intensity from the building is high; otherwise, the

Manuscript received May 2, 2012. This research was supported by a program of the 3rd ALOS Research Announcement, Japanese Aerospace Exploration Agency, and The Okawa Foundation for Information and Telecommunications.

M. Kajimoto is with the Department of Civil and Earth Resources Engineering, Kyoto University Graduate School of Engineering, Kyoto 615-8540, Japan (Tel./Fax: +81-75-383-3302; $\quad$ e-mail: kajimoto.muneyoshi.43m@st.kyoto-u.ac.jp).

J. Susaki is with the Department of Civil and Earth Resources Engineering, Kyoto University Graduate School of Engineering, Kyoto 615-8540, Japan (Tel./Fax: +81-75-383-3300; e-mail: susaki.junichi.3r@kyoto-u.ac.jp). scattering intensity is weak. This dependence of scattering on

the relative azimuth between the wall normal and the radar's ground range direction is a factor that makes land cover classification using SAR challenging.

The polarization orientation angle (POA) [1] can be used to reduce this dependence. Applying a four-component decomposition method [2], [3] to the fully polarimetric SAR data gives the surface scattering power (Ps), the double-bounce scattering power $(\mathrm{Pd})$, the volume scattering power $(\mathrm{Pv})$, and the helix scattering power (Pc); however, these components are also sensitive to POA. Thus, Yamaguchi et al. [4] proposed an algorithm that rotates the coherency matrix by the POA in order to reduce the dependence of the components on the relative azimuth. However, the dependence was found to remain even after this correction [5], and removing the remaining angular effects is considered nontrivial. Therefore, in this paper, we propose an algorithm for extracting urban areas from data containing these angular effects. The remainder of the paper is organized as follows. Section II explains the indices used in the proposed algorithm. The algorithm itself is then described in Section III, and experimental results are reported and discussed in Section IV. Finally, the paper is concluded in Section V.

\section{INDICES USED}

The format of polarimetric SAR data consists of a complex scattering matrix given by

$$
s=\left(\begin{array}{ll}
S_{H H} & S_{H V} \\
S_{V H} & S_{V V}
\end{array}\right)=\left(\begin{array}{ll}
a & c \\
c & b
\end{array}\right)
$$

Here, for simplicity, $\mathrm{S}_{\mathrm{HV}}$ and $\mathrm{S}_{\mathrm{VH}}$ are assumed to be equivalent [2], [3], and a coherency matrix expressed by

$$
\begin{gathered}
T=\left(\begin{array}{ccc}
T_{11} & T_{12} & T_{13} \\
T_{21} & T_{22} & T_{23} \\
T_{31} & T_{32} & T_{33}
\end{array}\right) \\
=\left(\begin{array}{ccc}
|a+b|^{2} & (a+b)(a-b)^{*} & 2(a+b) c^{*} \\
(a-b)(a+b)^{*} & |a-b|^{2} & 2(a-b) c^{*} \\
2 c(a+b)^{*} & 2 c(a-b)^{*} & 4|c|^{2}
\end{array}\right)
\end{gathered}
$$

\section{A. Four-component Decomposition}

Four-component decomposition is a method of decomposing the observed backscattering into four components, where each component is calculated from the coherency matrix. 
Furthermore a rotation can be applied to the coherency matrix as follows

$$
T=\left(\begin{array}{lll}
T_{11}(\theta) & T_{12}(\theta) & T_{13}(\theta) \\
T_{21}(\theta) & T_{22}(\theta) & T_{23}(\theta) \\
T_{31}(\theta) & T_{32}(\theta) & T_{33}(\theta)
\end{array}\right)=\left[R_{p}(\theta)\right] T\left[R_{p}(\theta)\right]^{\dagger} .
$$

Here, $\dagger$ denotes complex conjugation and transposition, and $R_{p}(\theta)$ is the rotation matrix given by

$$
\left[R_{p}(\theta)\right]=\left(\begin{array}{ccc}
1 & 0 & 0 \\
0 & \cos 2 \theta & \sin 2 \theta \\
0 & -\sin 2 \theta & \cos 2 \theta
\end{array}\right)
$$

\section{B. Polarization Orientation Angle (POA)}

POA, $\theta$, is estimated as follows:

$$
\theta=\frac{1}{4} \tan ^{-1} \frac{2 \operatorname{Re}\left(T_{23}\right)}{T_{22}-T_{33}},\left(-\frac{\pi}{4} \leq \theta \leq \frac{\pi}{4}\right)
$$

$\theta$ is determined such that the wall normal is parallel to the sensor's ground range direction and is equivalent to minimizing $T_{33}(\theta)$.

\section{METHODS}

For urban areas in Tokyo, Fig. 1 shows plots of the four power components and the total power (TP) versus POA before and after the scattering intensity was corrected according to the POA. The POA dependence can be seen to decrease as a result of the correction. After the correction, $\mathrm{Pv}$ is almost constant regardless of POA, whereas the other components still depend on POA. In general, the scattering intensity in urban areas is stronger than that in farmland. However, the scattering intensity in urban and mountain areas is similar in some cases. This similarity results from the POA dependence of urban areas. The scattering intensity from non-orthogonal buildings is weaker than that from orthogonal buildings. Hereinafter, this "orthogonality" condition denotes measurement geometry with POA of $0^{\circ}$. Discriminating between orthogonal urban areas and mountain areas is straightforward. However, the scattering intensity from non-orthogonal urban areas and mountain areas is similar. As a result, discriminating between these two land covers is hard in some cases. Therefore, instead of using the power components, another approach is necessary to discriminate between urban areas and mountain areas. As shown in Fig. 2, the proposed algorithm for urban area extraction consists of two steps. The first step is classification of urban/mountain area and farmland/bare ground/sea. The second step then classifies the urban and mountain areas.

\section{A. Classification Using Pv and TP}

Fig. 3 shows urban and farmland areas in Tokyo, Japan from which training data were obtained. Fig. 4 shows the results of principal component analysis (PCA) of $\mathrm{Pv}-\mathrm{TP}$ scattergrams for the training data areas, at POAs of $0^{\circ}, 15^{\circ}, 30^{\circ}$, and $45^{\circ}$. The

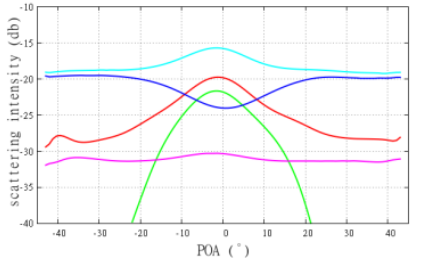

(a)

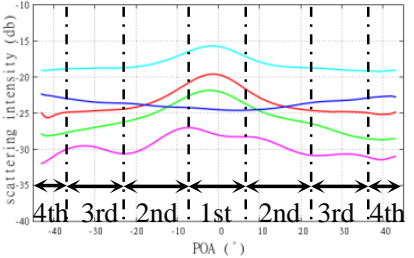

(b)
Fig. 1. Relation between POA and power components derived by four-component decomposition. (a) Uncorrected data, and (b) POA-corrected data. Red line denotes surface scattering power (Ps), green denotes double-bounce scattering power ( $\mathrm{Pd})$, navy blue denotes volume scattering power $(\mathrm{Pv})$, pink denotes helix scattering (Pc), and sky blue denotes total power (TP).

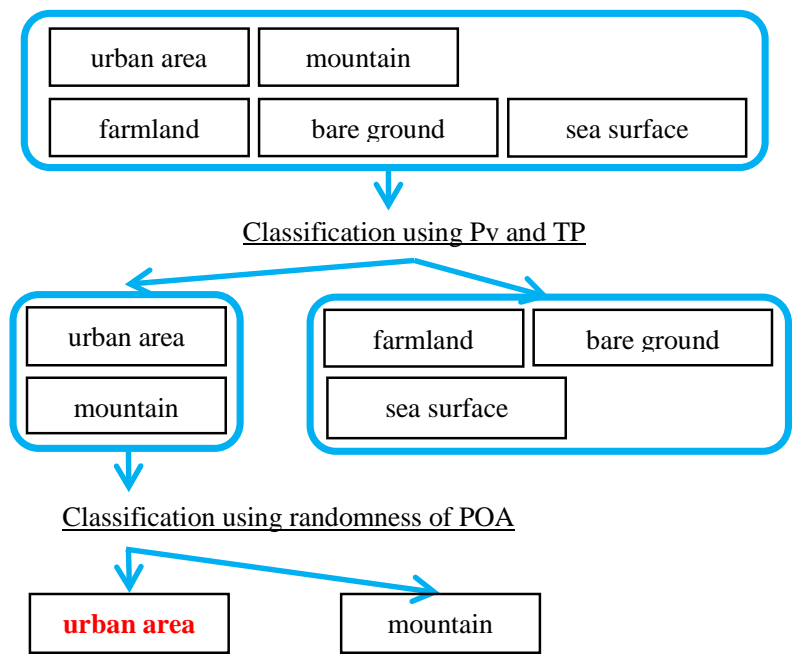

Fig. 2. Flowchart of urban area extraction.

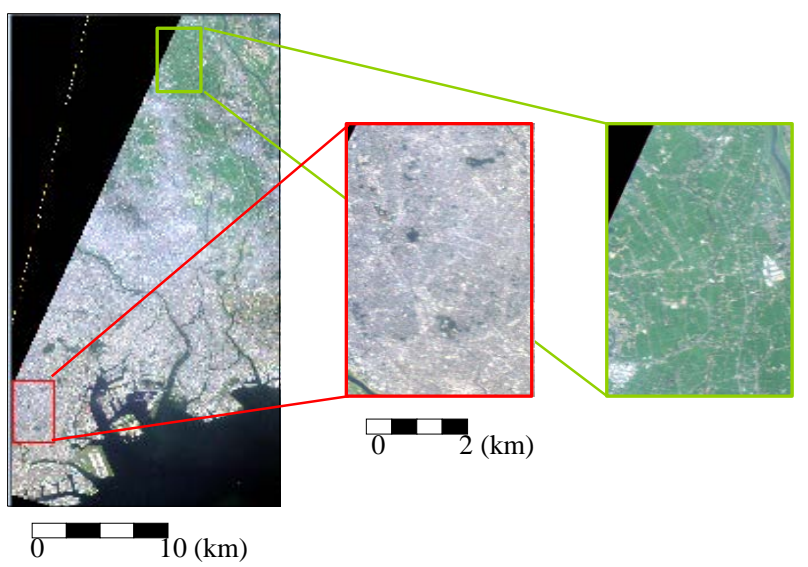

Fig. 3. Urban and farmland areas (Tokyo, Japan) from which training data were obtained.

combination of $\mathrm{Pv}$ and $\mathrm{TP}$ was found to be effective for discriminating between urban areas and farmland, and the scattergrams show that the discriminant between urban areas and farmland may depend on POA. We also attempted to utilize combinations of the other three power components (Ps, Pd, and Pc), but they were highly sensitive to POA and building type. Consequently, even within an urban area, these three scattering intensity parameters vary greatly. In contrast, $\mathrm{Pv}$ is not strongly dependent on POA, and TP is robust against differences in building type. Therefore, the combination of $\mathrm{Pv}$ and $\mathrm{TP}$ was 


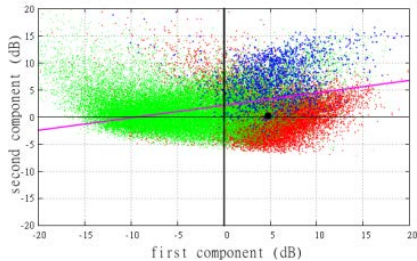

(a)

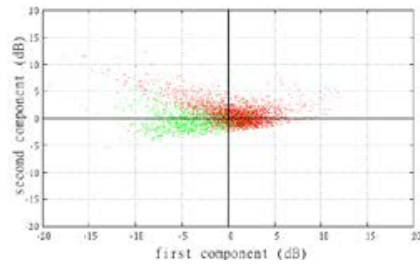

(c)

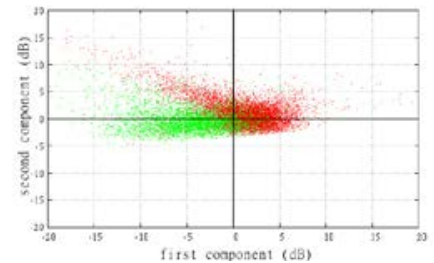

(b)

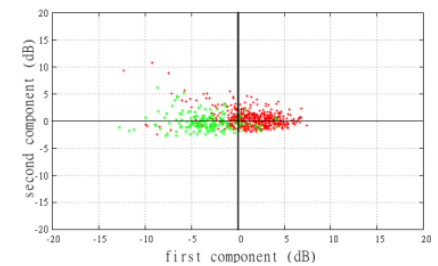

(d)
Fig. 4. Results of PCA of Pv-TP scattergrams for urban and farmland areas in Tokyo. Scattergrams at POA of (a) $0^{\circ}$, (b) $15^{\circ}$, (c) $30^{\circ}$, and (d) $45^{\circ}$. Horizontal and vertical axes denote the first and shifted second principal component axes (first threshold), respectively, and the pink line denotes the second threshold. Red, green, and navy points denote urban areas, farmland, and orthogonal farmland, respectively, and the black point denotes urban gravity.

found most suitable for the robust extraction of urban areas. Because the discriminant depends on POA, the POA space is divided into four categories: $\left(-7.5^{\circ}\right.$ to $\left.7.5^{\circ}\right),\left(-22.5^{\circ}\right.$ to $7.5^{\circ}, 7.5^{\circ}$ to $\left.22.5^{\circ}\right),\left(-37.5^{\circ}\right.$ to $-22.5^{\circ}, 22.5^{\circ}$ to $\left.37.5^{\circ}\right)$, and $\left(-45.0^{\circ}\right.$ to $-37.5^{\circ}, 37.5^{\circ}$ to $\left.45.0^{\circ}\right)$. Thus each pixel of a SAR image is assigned to one of these categories. In this paper, a scattering intensity with a negative POA is regarded as equivalent to that with the absolute value of its POA. This equivalency is considered acceptable because each component in Fig. 1 is approximately symmetrical about the POA of $0^{\circ}$.

Gravity points of urban areas and farmland were found to differ between areas. Therefore, the threshold used for land cover classification must be optimized for the study area of interest. However, in the proposed algorithm, manual threshold selection is reduced via the following procedure.

First, urban and farmland training data are manually selected from a study area. Next, principal component analysis is applied to the training data, and the threshold for discriminating between urban areas and farmland is determined. Initially, Otsu's method [6] was employed to determine the threshold for Tokyo. However, the resulting threshold was found to misclassify the majority of areas as urban. Hence, the threshold was determined from the means and standard deviations of the first principal components of the two land cover types. The standard deviation of the first principal components associated with farmland is larger than that of urban areas. As a result, when using the overall mean, the second principal component over-extracts urban areas in most cases. Therefore, the difference between the means of the two land cover types is divided in proportion to the standard deviations, and a break point is obtained. Then, the second principal component is shifted along the first principal component so as to pass the break point, and the shifted second principal component is used as the threshold. The threshold is then applied to another study area, and an attempt is made to separate the urban areas from farmland. The difference is calculated between the urban gravity points in the new area and in the initial study area, and this difference is used to adjust the threshold. The updated

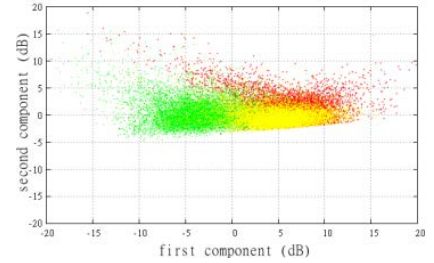

Fig. 5. Pv-TP scattergrams for urban, farmland, and mountain areas at POA of $15^{\circ}$ in Sapporo. Red, green, and yellow points denote urban, farmland, and mountain areas, respectively.

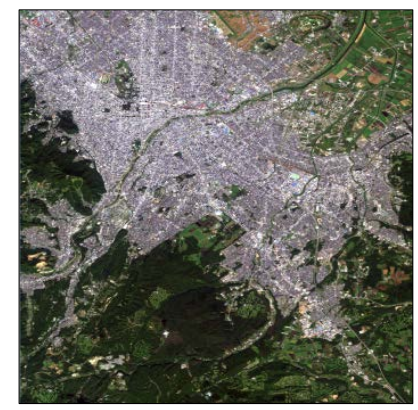

(a)

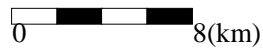

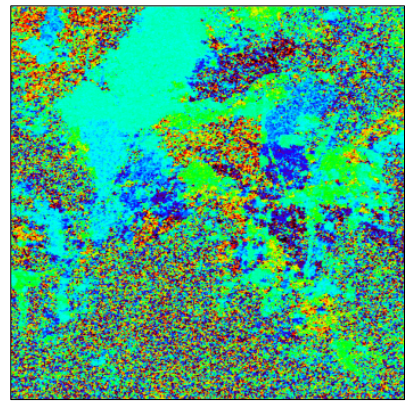

(b)

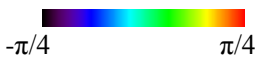

Fig. 6. POAs of pixels for urban and mountain areas in Sapporo: (a) AVNIR-2 image, and (b) POA map.

threshold is again applied to the new study area, and the gravity point difference is calculated. Iteration of this process is terminated when the change in the threshold is within a predefined limit.

Note that the scattering intensity from farmland orthogonal to the ground range direction is as strong as that from an urban area. Orthogonal farmland is seen in only the first POA category, and another discriminant is necessary to remove these data. In the proposed algorithm, a second discriminant is independently determined from the training data. This discriminant is a second threshold that is optimized after optimization of the first threshold. By applying the above procedure, land cover with weak scattering, sea, and bare ground are classified as farmland.

\section{B. Classification Using POA Randomness}

The Pv-TP scattergrams of urban areas and of mountain areas mostly overlap (Fig. 5). These two land covers cannot be clearly classified by setting a threshold as in Section III-A. However, the difference in POA randomness between urban and mountain areas is considered to be an appropriate index for classifying them. The majority of buildings in an urban area are aligned in the same direction, and thus, POAs in a district will be nearly homogenous; in contrast, mountain areas are covered by natural vegetation, and POAs differ randomly (Fig. 6). By considering this phenomenon, a procedure to discriminate between urban areas and mountain areas is proposed as follows.

1) Each pixel is labeled using one of five POA-based groups (Fig. 7(a)).

2) A window, denoted in red in Figure 7(b), is set surrounding a pixel. Taking each pixel in turn within the window, the 


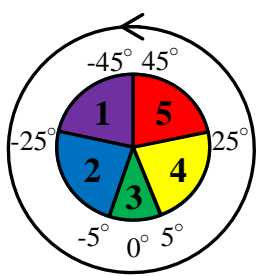

(a)

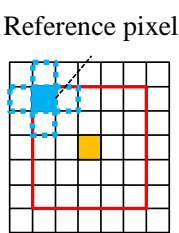

(b)

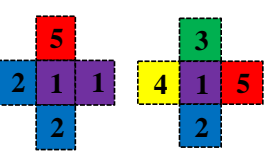

(c) (d)
Fig. 7. (a) Labeling based on POA, and (b), (c), (d) Assessment of POA randomness.

POA labels of the four neighboring pixels are compared with that of the central pixel, defined as the "reference pixel” (e.g., Label 1 in Fig. 7(d)). If all four pixels have labels that are equal to the reference pixel's label (Label 1) or are one away from it (Label 2 or 5), the pixel is not counted (Fig. 7(c)). In all other cases, the pixel is counted (Fig. 7(d)). The number of pixels counted is then assigned to the central pixel of the window (orange in Fig. 7(b)).

3) The center of the window is then shifted and step (2) is again executed.

Using this procedure, the pixel count is expected to be small in urban areas, and large in mountain areas.

At first, an attempt was made to classify urban and mountain areas by using a POA variation coefficient. However, the classification results were considered unacceptable, because the majority of non-orthogonal buildings were categorized as mountain areas. In Fig. 6, different POAs are randomly distributed in non-orthogonal urban areas. The accuracy of POA estimation decreases when POAs are far from $0^{\circ}$. Therefore, POA variation between neighboring pixels in non-orthogonal areas is as large as that in mountain areas, and discriminating between these two land covers using this method is not viable.

In this study, categorization using the POA-based groups explained above was instead applied. However, using too many or too few POA-based groups led to unsuccessful classification results. If the number of groups is small, POA randomness effects are increased and the categorization is poor. In contrast, if the number of groups is large, extraction of non-orthogonal urban areas is difficult. In non-orthogonal urban areas, the accuracy of POA estimation is low, thus POA values are somewhat different around reference pixels. POA randomness is large even in non-orthogonal urban areas, and little difference is found between non-orthogonal urban and mountain areas. Therefore, care must be taken that the number of POA-based groups is not too large. By considering the standard deviation of POAs in non-orthogonal urban areas (approximately $20^{\circ}$ ), five groups were deemed to be appropriate. In general, the number of pixels with POAs of around $0^{\circ}$ is much larger than that with POAs of around $45^{\circ}$ in the whole image when applying a filter to the coherency matrix (In this study, a $3 \times 3$ boxcar filter). Thus, each group's interval was decided as above, due the larger POA variation in mountain areas.

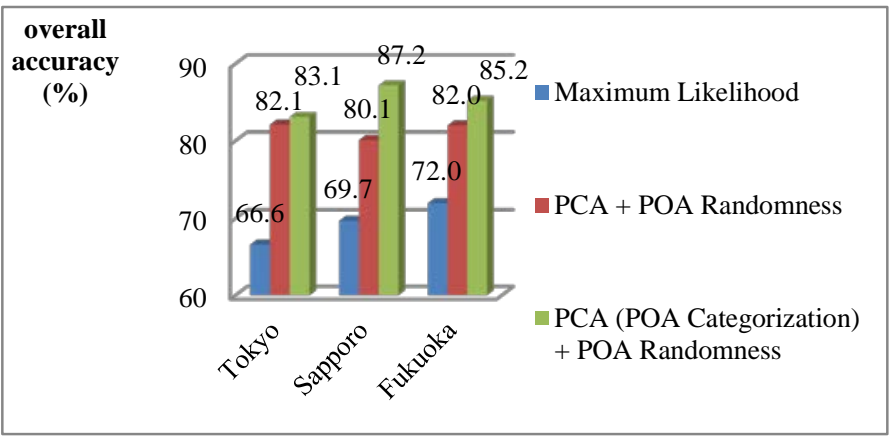

Fig. 8. Effect of POA categorization on accuracy for the three study areas.

\section{EXPERIMENTAL RESULTS AND DISCUSSION}

In this study, fully polarimetric Advanced Land Observing Satellite (ALOS)/Phased Array type L-band SAR (PALSAR) level 1.1 (L1.1) data were used. Furthermore, ALOS/Advanced Visible and Near Infrared Radiometer type 2 (AVNIR-2) optical sensor data were used as a reference. Three study areas were selected: Tokyo, Sapporo, and Fukuoka in Japan. Urban and farmland training data were manually selected from the Tokyo area. Thus, the threshold for discriminating between urban areas and farmland was determined for Tokyo, and then optimized for other areas.

To terminate the optimization loop, the minimum change in the urban gravity point was set to $0.01 \mathrm{~dB}$. If the minimum change is set smaller than $0.01 \mathrm{~dB}$, the number of iterations increases; however, the gravity point is almost the same as when the minimum is set to $0.01 \mathrm{~dB}$. For POA randomness calculations, the window size was set at $31 \times 31$ pixels, and the ratio between the pixel count and the total number of pixels in the window for discriminating between urban and mountain areas was set to 0.35 . The window size was determined such that a clear difference was seen between the POA randomness of non-orthogonal urban areas and mountain areas. If the window is small, this difference cannot be seen. If the window is too big, the boundary between urban and mountain areas becomes ambiguous. Moreover, the POA randomness threshold was set by ensuring that non-urban areas were not removed. If the POA randomness threshold is less than 0.35 , a proportion of non-urban areas are removed, whereas a threshold greater than 0.35 retains the majority of the mountain areas.

\section{A. Result of Classification Using Pv and TP}

Two issues were examined by conducting an accuracy assessment in all three study areas, where reference data were obtained manually (Fig. 8). The first is how the classification results of the proposed algorithm compare with those of a conventional (maximum likelihood) algorithm, and the second is the effect of POA categorization, described in Section III-A, on classification results. For comparison, urban area extraction was conducted using a conventional maximum likelihood estimation algorithm. Five training datasets were manually obtained a priori for urban areas, mountain areas, farmland, bare ground, and sea, in each study area. After training, maximum likelihood estimation was then applied to the Pv-TP scattergrams. In the calculation, the likelihood was inversely proportional to the Mahalanobis distance between the center of 


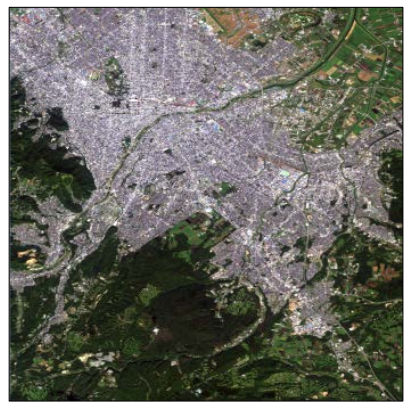

(a)

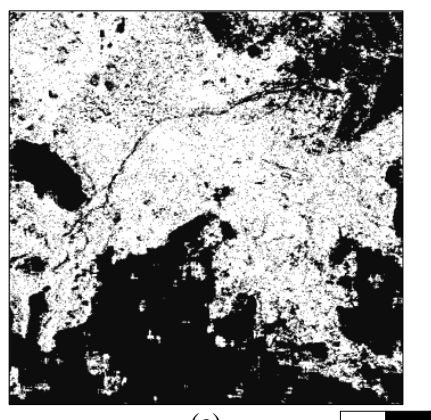

(c)

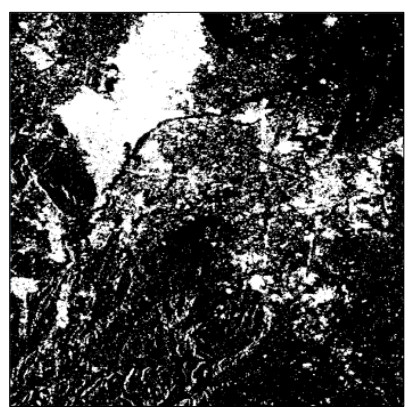

(b)

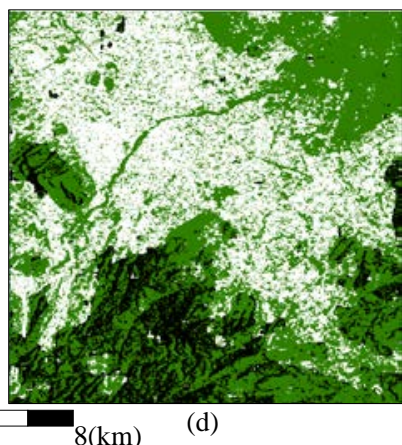

Fig. 9. Effect of POA categorization and POA randomness for Sapporo: (a) AVNIR-2 image; (b) classification generated by maximum likelihood estimation; (c) classification without POA categorization (white pixels denote urban areas and black pixels denote non-urban areas); (d) classification with POA categorization (white pixels denote urban areas, green pixels denote the pixels classified as farmland via procedure in Section III-A, and black pixels denote the pixels classified as mountain areas via procedure in Section III-B).

the training dataset and the target point. The results generated by maximum likelihood estimation were found to have lower accuracy than those generated by the proposed algorithm. Moreover, results generated by the proposed algorithm with POA categorization were found to have higher accuracy than those without it, especially in Sapporo where an improvement in classification accuracy of $7.1 \%$ was achieved. By optimizing the threshold for Tokyo to suit the other areas of interest in the PCA process, discriminant equations for each area were automatically estimated in the proposed algorithm.

Fig. 9 shows the results of classification without and with POA categorization for Sapporo. Most of the non-orthogonal urban areas were classified as mountain areas or bare ground by categorization using maximum likelihood estimation. In many case, non-orthogonal urban areas were included in land cover with weak scattering, which caused a great reduction in accuracy. Therefore, discriminating between non-orthogonal urban areas and mountain areas by using the power components is ineffective. Without categorization, urban areas were over-extracted. In contrast, urban and non-urban areas were classified more accurately by setting an appropriate threshold for each POA category. In heavily built-up urban areas, POA categorization is not as effective. However, categorization is valid in areas such as Sapporo with mixed land cover types. Classification using TP is successful in removing bare ground and mountains, but TP is sensitive to POA. Thus, POA categorization is applicable in these areas, and setting POA thresholds for each category enabled more accurate extraction of urban areas.

\section{B. Result of Classification Using POA Randomness}

Without the use of POA randomness, approximately $50 \%$ of mountain areas were misclassified as urban areas. Conversely, the addition of POA randomness succeeded in avoiding such misclassification (Fig. 9(d)).

Without POA randomness, pixels along mountain ridges were also misclassified as urban areas, because sections of the ridges have strong scattering intensity. The proposed algorithm properly classified such pixels as mountain areas.

\section{CONCLUSIONS}

In this paper, a classification algorithm was proposed that successfully extracts urban areas from polarimetric SAR images. $\mathrm{Pv}$ and $\mathrm{TP}$ data, generated by four-component decomposition, are used for classification. Pixels are categorized based on POAs and a different classification threshold is set for each category.

In general, the scattering intensity in urban areas is stronger than that in farmland. However, the scattering intensity depends on POA. The scattering intensity in non-orthogonal urban areas and that in orthogonal farmland is similar in some cases, which may lead to misclassification, such as the over-extraction of urban areas. Therefore, pixels with the same POAs should ideally be compared. In this paper, POA categorization reduced this misclassification.

Thus, POA randomness is able to discriminate urban areas from mountain areas, and experimental results demonstrate that the proposed method can extract urban areas in the study area. Moreover, the proposed algorithm automatically estimates the optimal POA threshold of other study areas from that determined for an initial study area. Classification results show that thresholds output by this algorithm are valid, and additional training data from images do not need to be analyzed.

Hence, the proposed algorithm for urban extraction is robust and applicable to various areas. The algorithm was applied to ALOS/PALSAR data, and application to higher resolution sensor data, for example, from TerraSAR-X, will be attempted in the future

\section{REFERENCES}

[1] H. Kimura, K.P. Papathanassiou, and I. Hajnsek, "Polarization orientation effects in urban areas on SAR data”, Proc. of the 2005 International Geosci. Remote Sens., pp.4863-4867, 2005.

[2] Y. Yamaguchi, T. Moriyama, M. Ishido, and H. Yamada, "Four-component scattering model for polarimetric SAR image decomposition”, IEEE Trans. Geosci. Remote Sens., vol. 43, no. 8, pp. 1699-1706, 2005.

[3] Y. Yamaguchi, Y. Yajima, and H. Yamada, "A Four-Component Decomposition of POLSAR Images Based on the Coherency Matrix", IEEE Geosci. Remote Sens. Letters, vol. 3, no. 3, pp. 292-296, 2006.

[4] Y. Yamaguchi, A. Sato, W. Boerner, R. Sato, and H. Yamada, "Four-Component Scattering Power Decomposition With Rotation of Coherency Matrix”, IEEE Trans. Geosci. Remote Sens., vol. 49, no 6, pp. 2251-2258, 2011.

[5] S. Iwasa and J. Susaki, "Classification of building area using azimuth angle and density indices derived from polarimetric SAR”, Proceedings of Joint Urban Remote Sensing Event, pp. 269-272, 2011.

[6] N. Otsu, "A threshold selection method from gray-level histograms". IEEE Trans. Sys., Man., Cyber., vol. 9, no. 1, pp. 62-66, 1979. 